\title{
Genetic diversity is a predictor of mortality in humans
}

Nathan A Bihlmeyer ${ }^{1,2}$, Jennifer A Brody ${ }^{35}$, Albert Vernon Smith ${ }^{32,33}$, Kathryn L Lunetta ${ }^{9,10}$, Mike Nalls ${ }^{6}$, Jennifer A Smith ${ }^{14}$, Toshiko Tanaka ${ }^{36}$, Gail Davies ${ }^{15,16}$, Lei Yu ${ }^{18}$, Saira Saeed Mirza ${ }^{21}$, Alexander Teumer ${ }^{27,28,}$ Josef Coresh ${ }^{38}$, James S Pankow ${ }^{39}$, Nora Franceschini ${ }^{40}$, Anish Scaria ${ }^{3}$, Junko Oshima ${ }^{4}$, Bruce M Psaty ${ }^{5}$, Vilmundur Gudnason ${ }^{32,33}$, Gudny Eiriksdottir ${ }^{32}$, Tamara B Harris ${ }^{34}$, Hanyue Li ${ }^{9}$, David Karasik ${ }^{12}$, Douglas P Kiel ${ }^{12}$, Melissa Garcia ${ }^{7}$, Yongmei Liu ${ }^{8}$, Jessica D Faul ${ }^{13}$, Sharon LR Kardia ${ }^{14}$, Wei Zhao ${ }^{14}$, Luigi Ferrucci ${ }^{36}$, Michael Allerhand ${ }^{15}$, David C Liewald ${ }^{15}$, Paul Redmond ${ }^{16}$, John M Starr ${ }^{15,17}$, Philip L De Jager ${ }^{19}$, Denis A Evans ${ }^{20}$, Nese Direk ${ }^{21}$, Mohammed Arfan Ikram 21,22,23, André Uitterlinden ${ }^{21,26}$, Georg Homuth ${ }^{27}$, Roberto Lorbeer ${ }^{28}$, Hans J Grabe ${ }^{29,30}$, Lenore Launer ${ }^{34}$, Joanne M Murabito ${ }^{10,11}$, Andrew B Singleton ${ }^{6}$, David R Weir ${ }^{13}$, Stefania Bandinelli ${ }^{37}$, lan J Deary ${ }^{15,16}$, David A Bennett ${ }^{18}$, Henning Tiemeier ${ }^{21,24,25}$, Thomas Kocher ${ }^{31}$, Thomas Lumley ${ }^{3^{*}}$ and Dan E Arking ${ }^{2^{*}}$

\section{Abstract}

Background: It has been well-established, both by population genetics theory and direct observation in many organisms, that increased genetic diversity provides a survival advantage. However, given the limitations of both sample size and genome-wide metrics, this hypothesis has not been comprehensively tested in human populations. Moreover, the presence of numerous segregating small effect alleles that influence traits that directly impact health directly raises the question as to whether global measures of genomic variation are themselves associated with human health and disease.

Results: We performed a meta-analysis of 17 cohorts followed prospectively, with a combined sample size of 46,716 individuals, including a total of 15,234 deaths. We find a significant association between increased heterozygosity and survival $(P=0.03)$. We estimate that within a single population, every standard deviation of heterozygosity an individual has over the mean decreases that person's risk of death by $1.57 \%$.

Conclusions: This effect was consistent between European and African ancestry cohorts, men and women, and major causes of death (cancer and cardiovascular disease), demonstrating the broad positive impact of genomic diversity on human survival.

Keywords: Heterozygosity, Human, Survival, GWAS

\footnotetext{
* Correspondence: t.lumley@auckland.ac.nz; arking@jhmi.edu

${ }^{3}$ Department of Statistics, University of Auckland, 303.325 Science Centre, Private Bag 92019, Auckland 1142, New Zealand

${ }^{2}$ McKusick-Nathans Institute of Genetic Medicine, Johns Hopkins University

School of Medicine, BRB Room 447, 733 N. Broadway St, Baltimore, MD

21205, USA

Full list of author information is available at the end of the article
} 


\section{Background}

With the advent of genome-wide association studies (GWAS), and more recently whole-exome and wholegenome sequencing, remarkable progress has been made in elucidating the genetics of complex traits, with numerous genetic variants each explaining a small fraction of the variance $[1,2]$. The presence of numerous segregating small effect alleles within the genome that influence traits that directly impact health raises the question of whether global measures of genomic variation are themselves associated with human health and disease. Indeed, increased fitness has been associated with the increase of genetic diversity across many organisms [3,4], including humans [5-8], and is often referred to as positive Heterozygosity Fitness Correlations (HFCs). In particular, associations have been found between heterozygosity at the Major Histocompatibility Complex (MHC) (a.k.a. Human Leukocyte Antigen, HLA) region and general health in humans [9]. In the case of heterozygosity in the $\mathrm{MHC}$ region, the cause of a positive HFC being observed is believed to be the result of increased antibody diversity conveying robust pathogen resistance and therefore increased general health [10]. However, in the case of increased whole-genome heterozygosity, the mechanism of action is less readily apparent. Two general mechanisms that act at a genome level to influence fitness have been proposed. The first is compensation for recessive deleterious mutations [11], whereas the second is a specific advantage of the heterozygous state over either homozygous state (overdominance/heterozygous advantage) [11], such as that observed for the sickle cell mutation in the presence of endemic malarial disease. It has been proposed that compensation for deleterious mutations occurs at many loci and is the major mechanism at work in HFCs, with overdominance occurring at few loci but with greater effect size per occurrence [11].

\section{Results and discussion}

Various heterozygosity metrics have been proposed [12]. The heterozygosity metric used in this study is the sum of all heterozygous loci divided by the expected state given the allele frequency under Hardy-Weinberg Equilibrium $t=\frac{\sum_{0 ; 1}}{\sum^{2 p(1-p)}}$ : where $\mathrm{p}$ is the frequency of the major allele in each cohort. This metric up-weights loci where the expectation of being heterozygous is low. Given the relationship between effect size and allele frequency $[13,14]$, up-weighting loci with low minor allele frequencies should maximize the ability to detect a HFC in humans under a model in which the compensation for deleterious alleles is the major mechanism driving HFCs. Only Single Nucleotide Polymorphisms (SNPs) on the autosomes were considered.
To test for the effect of genome-wide heterozygosity on survival, we performed a meta-analysis of 17 cohorts (13 European ancestry, 4 African American ancestry) followed prospectively, with a combined sample size of 46,716 individuals, including a total of 15,234 deaths (Additional file 1: Table S1). Within each cohort, a Cox proportional hazards model (CoxPH) was used comparing age at study entry to age at study exit (death) or most recent follow-up (alive), and included covariates known to affect survival (sex, highest education level, Body Mass Index (BMI), income level, center where DNA was collected, and the first ten principal components to adjust for population substructure). Since each cohort used a different number of SNPs (Additional file 1: Table $\mathrm{S} 1$ ), the variances of the heterozygosity metrics are not the same (they are dependent on the total number of SNPs in the metric), and effect sizes from each cohort are not directly comparable. Using Stouffer's method to combine Zscores, weighted by the number of deaths in each cohort, we find a significant association between increased heterozygosity and survival $(P=0.03)$. To assess effect size, we standardized the beta estimates by multiplying them by the standard deviation of the heterozygosity metric for each cohort [15]. This method does not completely account for the aforementioned bias; however, it is the most appropriate method to determine an interpretable effect size. Combining the standardized beta estimates using inverse variance weighting demonstrates that for every standard deviation increase in heterozygosity a person has over the population mean, they are expected to have a $1.57 \%$ decreased risk of death (Figure 1). There was no evidence for heterogeneity across studies, and a direct comparison of European Ancestry to African ancestry cohorts showed no significant difference (Figure 2, $\mathrm{P}=0.80$ ); thus, all downstream analyses combined European and African ancestry cohorts.

To test whether all chromosomes are contributing equally to the association between heterozygosity and survival, each study subject's heterozygosity score was recalculated using only SNPs from a given chromosome. An inverse-variance meta-analysis for each chromosome was performed across studies, followed by a meta-analysis of the chromosomal results (Figure 3). No significant difference was observed between effects across chromosomes $(P=0.17)$. To test whether all major causes of death contribute equally to our genome-wide finding, death caused by cancer, death caused by CVD, and other causes of death were each analyzed separately. A meta-analysis for each cause of death was performed as described above, followed by a test for heterogeneity and model fitting. Our results demonstrate that heterozygosity is protective for all causes of death, with no significant evidence for heterogeneity (Figure 4, $\mathrm{P}=0.79$ ). To assess if heterozygosity levels impact women differently from men, meta-analyses 


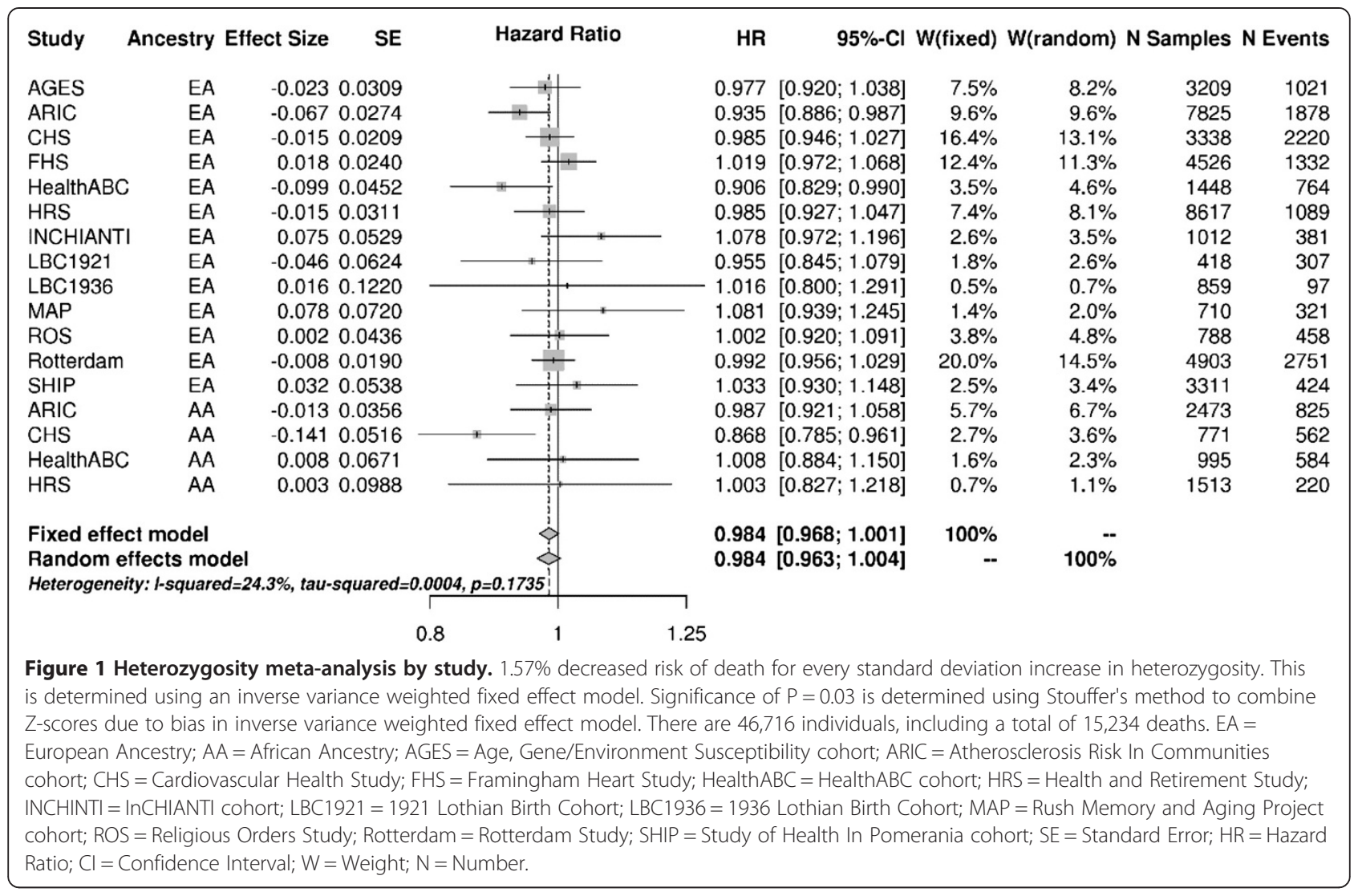

were performed separately for each sex. Our results do not provide evidence for a differential effect of heterozygosity on survival in men vs. women (Figure $5, \mathrm{P}=0.49$ ).

\section{Conclusions}

In summary, this study provides evidence that the protective effect of increased heterozygosity seen in lower organisms functions in humans as well and may have implications for how we design future studies to identify genetic determinants of human disease and survival. We estimate that within a single population, every standard deviation of heterozygosity an individual has over the mean decreases that person's risk of death by $1.57 \%$. Interestingly, this seems to be true even if the population itself has reduced mean heterozygosity. In future studies, limiting to heterozygosity in proximity to genes and/or regulatory elements may reveal if some regions are more sensitive to heterozygosity than others. Increasing the African ancestry sample size may increase power to see a difference between ancestry groups. Overall the consistency we observed between European and African ancestry, males and females, and major causes of death demonstrate a broad positive impact of genomic diversity on human survival.

\section{Methods}

Methods for each individual cohort can be found in Additional file 2: Text S1. Self-described Caucasian ("white", "Caucasian") and African ancestry ("black", "African American") individuals were included after excluding first and second degree relatives and genetic outliers. Genetic outliers were defined by merging genotyping data with HapMap3 data, and calculating the Euclidean distance from a combined reference HapMap3 population

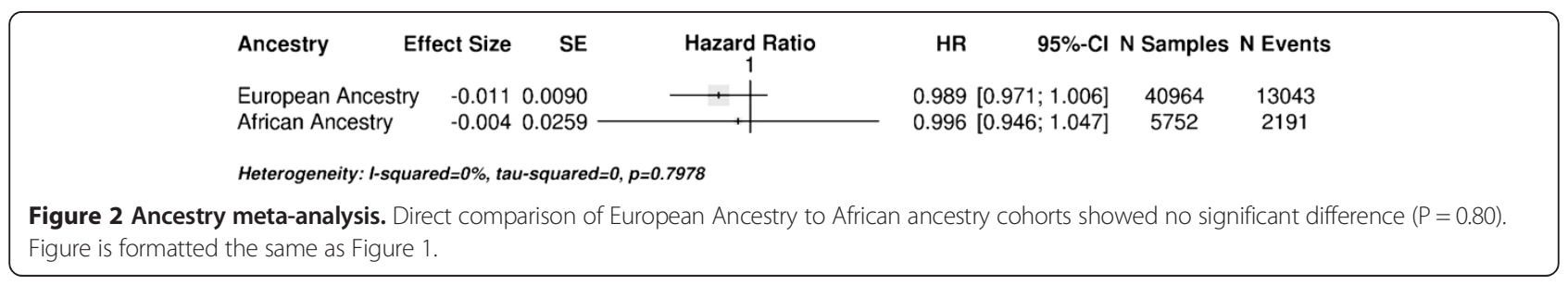




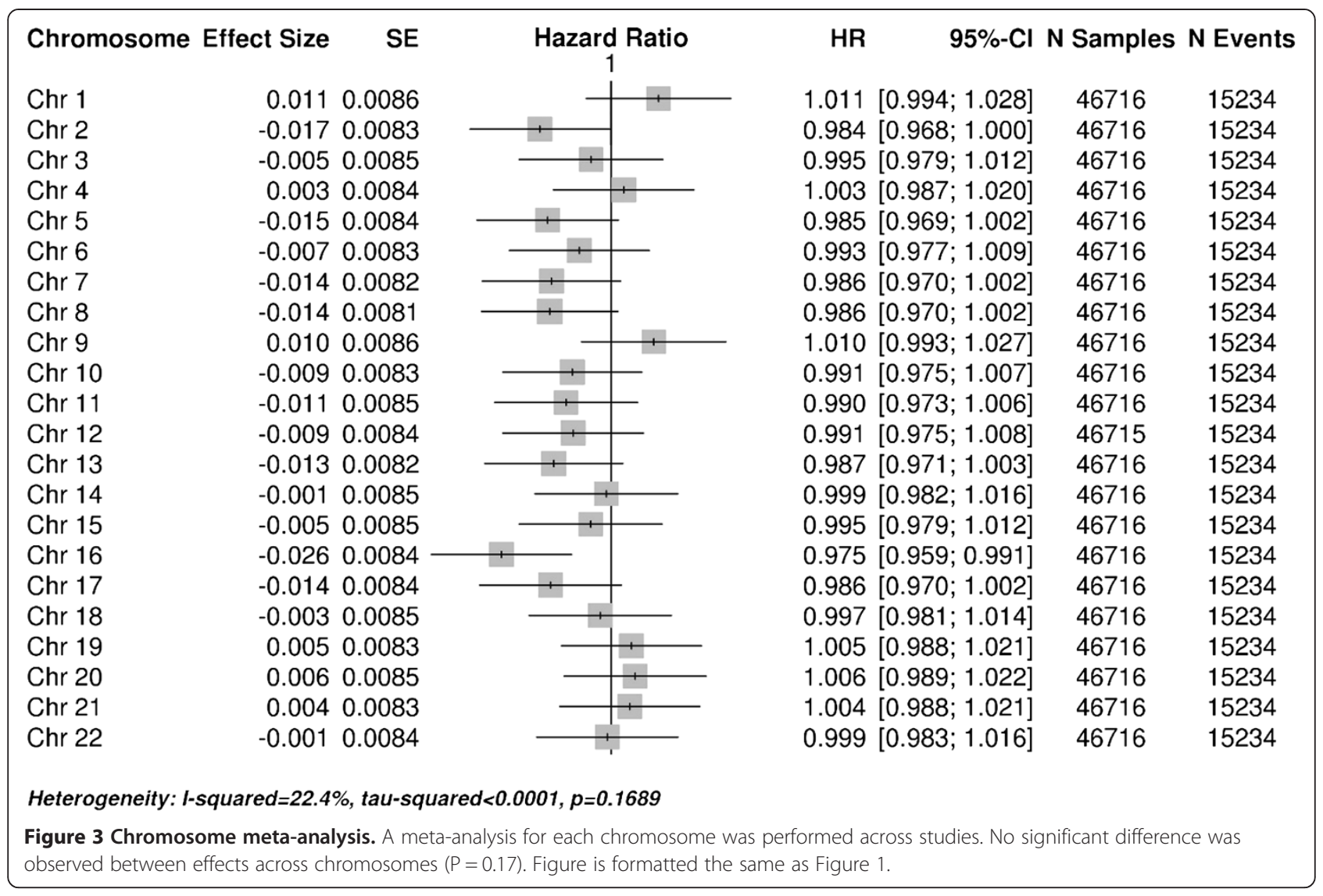

$($ Caucasian $=\mathrm{CEU}+\mathrm{TSI}$, African ancestry $=\mathrm{ASW}+\mathrm{YRI}+$ MKK + LWK) cluster centroid in the first 3 PC space weighted by explained variance. Specifically, the standard deviation of Euclidean distance was determined for each HapMap reference group, and any sample greater than ten standard deviations away from centroid were defined as genetic outliers and excluded.

Directly genotyped SNPs were used for all analyses (Additional file 3: Figure S1). Imputed SNPs were not used to avoid issues with genotype accuracy and bias towards the reference panel. SNP exclusion criteria included: monomorphic in the dataset, non-unique mapping to Hg19, SNPs which are no longer in the company provided annotation file for the SNP array, $>0.5 \%$ missing data, MAF $\leq 10 \%$, HWE p-value $\geq 0.001$, and non-autosomal SNPs. The heterozygosity metric is the sum of all heterozygous loci divided by the expected state given the allele frequency under Hardy-Weinberg Equilibrium: $t=\frac{\sum_{0 ; 1}}{\sum^{2 p(1-p)}}$ where $\mathrm{p}$ is the frequency of the major allele.

Separate association analyses were run for Caucasian and African ancestry samples from each cohort. The Cox Proportional Hazard Model (CoxPH) included covariates for Body Mass Index (BMI) at first visit and first ten principal components, and the 'strata' function for sex, education level (defined as $1 . \leq 11$ th grade, 2 . high school diploma, general equivalence diploma or some vocational school, 3. 1-4 years of college, 4. Some graduate/professional school, and Missing), income level (defined by cohorts), and center of DNA collection within cohorts. The CoxPH model was set up so that the outcome was age at study entry, age at study exit, and a binary variable coding state of death (1: Dead, 0: Alive). Age is measured in units of years, but is accurate to the nearest day.

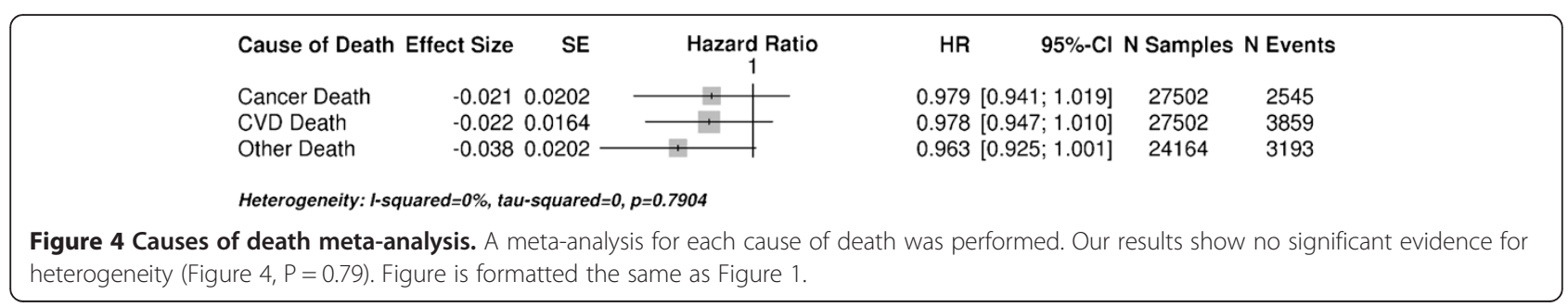




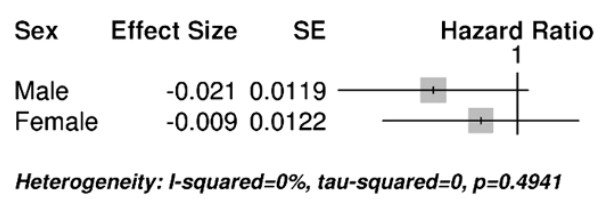

\begin{tabular}{|c|c|c|}
\hline $95 \%-\mathrm{Cl}$ & N Samples & N Events \\
\hline $.980[0.957 ; 1.003]$ & 20508 & 759 \\
\hline $0.991[0.968 ; 1.015]$ & 26258 & 7636 \\
\hline
\end{tabular}

Figure $\mathbf{5}$ Sex meta-analysis. A meta-analysis was performed separately for each sex. Our results do not provide evidence for a differential effect of heterozygosity on survival in men vs. women (Figure 5, P=0.49). Figure is formatted the same as Figure 1.

For the meta-analysis, significance was determined by Stouffer's method [16] calculated as a two-sided test by incorporating Z-scores derived from two-sided tests performed in each cohort. We standardized the beta estimates by multiplying them by the standard deviation of the heterozygosity metric for each cohort, to account for the fact that the effect size is proportional to the variance in the heterozygosity metric. The variance heterozygosity metric in turn is proportional to the inverse of the square root of the number of SNPs used to determine the heterozygosity metric. Because most cohorts used different genotyping arrays, a large bias is introduced into the meta-analysis. Stouffer's method completely removes this bias; however, cannot estimate a combined effect size, only the overall significance. To get an estimate of the combined effect size (recognizing that the P-value and associated confidence intervals will be inflated), we used inverse variance weighting of the standardized cohort effect sizes, which partially corrects the bias and allows for the combined effect size to be estimated.

\section{Ethics statements}

Institutional Review Board approvals were obtained by each participating ARIC study center (the Universities of NC, MS, MN, and John Hopkins University) and the coordinating center (University of $\mathrm{NC}$ ), and the research was conducted in accordance with the principles described in the Helsinki Declaration. All subjects in the ARIC study gave informed consent. For more information see dbGaP Study Accession: phs000280.v2.p1. JHSPH IRB number H.34.99.07.02.A1. Manuscript proposal number MS1964.

HealthABC Human subjects protocol UCSF IRB is H5254-12688-11.

CHS was approved by institutional review committees at each site, the subjects gave informed consent, and those included in the present analysis consented to the use of their genetic information for the study of cardiovascular disease. It is the position of the UW IRB that these studies of de-identified data, with no patient contact, do not constitute human subjects research. Therefore we have neither an approval number, nor an exemption.

IRB permission to conduct genetics-related work in the Health and Retirement Study (HRS) is granted under the project title, "Expanding a National Resource for Genetic Research in Behavioral \& Health Science" (HUM00063444). The IRB that approved this project is the Health Sciences and Behavioral Sciences Institutional Review Board at the University of Michigan. No manuscript proposal is required for use of HRS data.

Inchianti ethics review statement: The study protocol was approved by the Italian National Institute of Research and Care of Aging Institutional Review and Medstar Research Institute (Baltimore, MD).

The Religious Orders Study (ORA\# 91020181) and the Rush Memory and Aging Project (ORA\# 86121802) were approved by the Institutional Review Board of Rush University Medical Center. Written informed consent was obtained from all the participants.

The SHIP study followed the recommendations of the Declaration of Helsinki. The study protocol of SHIP was approved by the medical ethics committee of the University of Greifswald. Written informed consent was obtained from each of the study participants. The SHIP study is described in PMID: 20167617.

The Rotterdam Study has been approved by the medical ethics committee according to the Population Study Act Rotterdam Study, executed by the Ministry of Health, Welfare and Sports of the Netherlands. A written informed consent was obtained from all participants.

The Boston University Medical Campus Institutional Review Board approved the FHS genome-wide genotyping (protocol number $\mathrm{H}$-226671) and genetic investigation of aging and longevity phenotypes (protocol number $\mathrm{H}-24912$ ).

The Age, Gene/Environment Susceptibility Reykjavik Study has been funded by NIH contract N01-AG-12100, the NIA Intramural Research Program, Hjartavernd (the Icelandic Heart Association), and the Althingi (the Icelandic Parliament). The study is approved by the Icelandic National Bioethics Committee, (VSN: 00063) and the Data Protection Authority. The researchers are indebted to the participants for their willingness to participate in the study.

Ethics permission for the LBC studies was obtained from the Multi-Centre Research Ethics Committee for Scotland (MREC/01/0/56) and from Lothian Research Ethics Committee (LBC1936: LREC/2003/2/29 and LB 
C1921: LREC/1998/4/183). The research was carried out in compliance with the Helsinki Declaration. All subjects gave written, informed consent.

\section{Additional files}

Additional file 1: Table S1. Descriptive breakdown of each cohort and summary statistics.

Additional file 2: Text S1. Additional Methods for each individual cohort. Additional file 3: Figure S1. Heterozygosity Metrics Determined Using Different SNP Lists. The dataset used was genome wide SNP data from sequencing of 503 individuals with European ancestry from $1000 \mathrm{G}$ phase 3 release. The SNP lists used were: 1) all SNPs 2) SNPs on the Illumina $1 \mathrm{M}$ 3) SNPs on the Illumina 610quad 4) SNPs on the Illumina Omni2.5 and 5) SNPs on the Affymetrix 6.0. This is to determine if SNP selection on the arrays biases the heterozygosity metric. We see high correlation and no systematic bias.

\section{Competing interests}

The authors declare that they have no competing interests.

\section{Authors' contributions}

Designed Study: NAB, TL, and DEA. Ran Analyses: NAB, JAB, AVS, KLL, MN JAS, TT, GD, LY, SSM, AT. Contributed Data: JC, JSP, NF, AS, JO, BMP, VG, GE, $\mathrm{TBH}, \mathrm{HL}, \mathrm{DK}, \mathrm{DPK}, \mathrm{MG}, \mathrm{YL}, J \mathrm{DF}, \mathrm{SLRK}, \mathrm{WZ}, \mathrm{LF}, \mathrm{MA}, \mathrm{DCL}, \mathrm{PR}, J \mathrm{MS}, \mathrm{PLD}, \mathrm{DAE}, \mathrm{ND}$, MAI, AU, GH, RL, HJG, LL, JMM, ABS, DRW, SB, IJD, DAB, HT, TK, TL, DEA. Al authors read and approved the final manuscript.

\section{Acknowledgements}

\section{Funding}

Funded in part by training grant (NIGMS) 5T32GM07814.

This material is based upon work supported by the National Science Foundation Graduate Research Fellowship under Grant No. DGE-1232825. Any opinion, findings, and conclusions or recommendations expressed in this material are those of the authors(s) and do not necessarily reflect the views of the National Science Foundation.

\section{Cohorts}

\section{ARIC}

The Atherosclerosis Risk in Communities Study is carried out as a collaborative study supported by National Heart, Lung, and Blood Institute contracts (HHSN268201100005C, HHSN268201100006C, HHSN268201100007C, HHSN268201100008C, HHSN268201100009C, HHSN268201100010C, HHSN268201100011C, and HHSN268201100012C), R01HL087641, R01HL59367 and R01HL086694; National Human Genome Research Institute contract U01HG004402; and National Institutes of Health contract HHSN268200625226C. The authors thank the staff and participants of the ARIC study for their important contributions. Infrastructure was partly supported by Grant Number UL1RR025005, a component of the National Institutes of Health and NIH Roadmap for Medical Research.

\section{AGES}

The Age, Gene/Environment Susceptibility Reykjavik Study has been funded by NIH contract N01-AG-12100, the NIA Intramural Research Program, Hjartavernd (the Icelandic Heart Association), and the Althingi (the Icelandic Parliament). The study is approved by the Icelandic National Bioethics Committee, (VSN: 00-063) and the Data Protection Authority. The researchers are indebted to the participants for their willingness to participate in the study. $\mathrm{CHS}$

Cardiovascular Health Study: This CHS research was supported by NHLBI contracts HHSN268201200036C, HHSN268200800007C, N01HC55222,

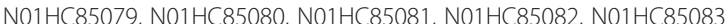
N01HC85086; and NHLBI grants HL080295, HL087652, HL105756, HL085251 with additional contribution from the National Institute of Neurological Disorders and Stroke (NINDS). Additional support was provided through AG023629 from the National Institute on Aging (NIA). A full list of principal CHS investigators and institutions can be found at CHS-NHLBI.org/.
The provision of genotyping data was supported in part by the National Center for Advancing Translational Sciences, CTSI grant UL1TR000124, and the National Institute of Diabetes and Digestive and Kidney Disease Diabetes Research Center (DRC) grant DK063491 to the Southern California Diabetes Endocrinology Research Center.

The content is solely the responsibility of the authors and does not necessarily represent the official views of the National Institutes of Health. FHS

Funding: The Framingham Heart Study analyses were supported by the National Institute of Aging (R01AG29451). This research was conducted in part using data and resources from the Framingham Heart Study of the National Heart Lung and Blood Institute of the National Institutes of Health and Boston University School of Medicine. The analyses reflect intellectual input and resource development from the Framingham Heart Study investigators participating in the SNP Health Association Resource (SHARe) project. This work was partially supported by the National Heart, Lung and Blood Institute's Framingham Heart Study (Contract No. N01-HC-25195) and its contract with Affymetrix, Inc for genotyping services (Contract No. N02-HL-6-4278). A portion of this research utilized the Linux Cluster for Genetic Analysis (LinGA-II) funded by the Robert Dawson Evans Endowment of the Department of Medicine at Boston University School of Medicine and Boston Medical Center. Dr. Kiel was partially supported by the National Institute of Arthritis Musculoskeletal and Skin Diseases (R01 AR41398).

\section{HealthABC}

This research was supported by NIA contracts N01AG62101, N01AG62103, and N01AG62106 and was supported in part by the Intramural Research Program of the NIH, National Institute on Aging (Z01 AG000949-02 and Z01 AG007390-07, Human subjects protocol UCSF IRB is H5254-12688-11). The genome-wide association study was funded by NIA grant 1R01AG032098 01A1 to Wake Forest University Health Sciences and genotyping services were provided by the Center for Inherited Disease Research (CIDR). CIDR is fully funded through a federal contract from the National Institutes of Health to The Johns Hopkins University, contract number HHSN268200782096C. This study utilized the high-performance computational capabilities of the Biowulf Linux cluster at the National Institutes of Health, Bethesda, Md. (http://biowulf.nih.gov).

HRS

HRS is supported by the National Institute on Aging (NIA U01AG009740). The genotyping was funded separately by the National Institute on Aging (RC2 AG036495, RC4 AG039029). Our genotyping was conducted by the NIH Center for Inherited Disease Research (CIDR) at Johns Hopkins University. Genotyping quality control and final preparation of the data were performed by the Genetics Coordinating Center at the University of Washington. InCHIANTI

The InCHIANTI study baseline (1998-2000) was supported as a "targeted project" (ICS110.1/RF97.71) by the Italian Ministry of Health and in part by the U.S. National Institute on Aging (Contracts: 263 MD 9164 and 263 MD 821336)

LBC

Lothian Birth Cohorts 1921 and 1936 (LBC1921, LBC1936)

We thank the cohort participants and team members who contributed to these studies. Phenotype collection in the Lothian Birth Cohort 1921 was supported by the BBSRC, The Royal Society and The Chief Scientist Office of the Scottish Government. Phenotype collection in the Lothian Birth Cohort 1936 was supported by Age UK (The Disconnected Mind project). Genotyping of the cohorts was funded by the UK Biotechnology and Biological Sciences Research Council (BBSRC). The work was undertaken by The University of Edinburgh Centre for Cognitive Ageing and Cognitive Epidemiology, part of the cross council Lifelong Health and Wellbeing Initiative (MR/K026992/1). Funding from the BBSRC, and Medical Research Council (MRC) is gratefully acknowledged.

\section{MAP/ROS}

The MAP and ROS data used in this analysis was supported by National Institute on Aging grants P30AG10161, R01AG17917, R01AG15819,

R01AG30146, the Illinois Department of Public Health, and the Translational Genomics Research Institute.

\section{Rotterdam}

The Rotterdam Study is supported by Erasmus Medical Centre and Erasmus University Rotterdam, the Netherlands Organization for Scientific Research (NWO), the Netherlands Organization for Health Research and Development (ZonMw), the Research Institute for Diseases in the Elderly (RIDE), the 
Netherlands Genomics Initiative, the Ministry of Education, Culture and Science, the Ministry of Health, Welfare and Sports, the European Commission (DG XII), and the Municipality of Rotterdam. Prof. Tiemeier was supported by the VIDI grant of ZonMw (2009-017.106.370). Dr. Ikram was supported by the VENI grant of NWO. The funders had no role in the study design or data collection and analysis.

SHIP

SHIP is part of the Community Medicine Research net of the University of Greifswald, Germany, which is funded by the Federal Ministry of Education and Research (grants no. 01ZZ9603, 01ZZ0103, and 01ZZ0403), the Ministry of Cultural Affairs as well as the Social Ministry of the Federal State of Mecklenburg-West Pomerania, and the network 'Greifswald Approach to Individualized Medicine (GANI_MED)' funded by the Federal Ministry of Education and Research (grant 03IS2061A). Genome-wide data have been supported by the Federal Ministry of Education and Research (grant no. 03ZIK012) and a joint grant from Siemens Healthcare, Erlangen, Germany and the Federa State of Mecklenburg- West Pomerania. The University of Greifswald is a member of the 'Center of Knowledge Interchange' program of the Siemens AG and the Caché Campus program of the InterSystems $\mathrm{GmbH}$.

\section{Author details}

'Predoctoral Training Program in Human Genetics, McKusick-Nathans Institute of Genetic Medicine, Johns Hopkins University School of Medicine, Baltimore, MD, USA. ${ }^{2}$ McKusick-Nathans Institute of Genetic Medicine, Johns Hopkins University School of Medicine, BRB Room 447, 733 N. Broadway St, Baltimore, MD 21205, USA. ${ }^{3}$ Department of Statistics, University of Auckland, 303.325 Science Centre, Private Bag 92019, Auckland 1142, New Zealand. ${ }^{4}$ Department of Pathology, University of Washington, Seattle, WA, USA. ${ }^{5}$ Departments of Medicine, Epidemiology, and Health Services, University of Washington, Seattle, WA, USA. ${ }^{6}$ Laboratory of Neurogenetics, National Institute on Aging, National Institutes of Health, Bethesda, MD, USA

${ }^{7}$ Laboratory of Epidemiology, Demography and Biometry, National Institute on Aging, National Institutes of Health, Bethesda, MD, USA. ${ }^{8}$ Department of Epidemiology and Prevention, Division of Public Health Sciences, Wake Forest University School of Medicine, Winston-Salem, NC, USA. ${ }^{9}$ Department of Biostatistics, Boston University School of Public Health, Boston, MA, USA. ${ }^{10}$ The National Heart Lung and Blood Institute's Framingham Heart Study, Framingham, MA, USA. ${ }^{11}$ Section of General Internal Medicine, Department of Medicine, Boston University School of Medicine, Boston, MA, USA.

${ }^{12}$ Institute for Aging Research, Hebrew Senior Life, Department of Medicine, Beth Israel Deaconess Medical Center and Harvard Medical School, Cambridge, MA, USA. ${ }^{13}$ Survey Research Center, Institute for Social Research, University of Michigan, Ann Arbor, MI, USA. ${ }^{14}$ Department of Epidemiology, School of Public Health, University of Michigan, Ann Arbor, MI, USA. ${ }^{15}$ Centre for Cognitive Ageing and Cognitive Epidemiology, The University of Edinburgh, Edinburgh, UK. ${ }^{16}$ Department of Psychology, The University of Edinburgh, Edinburgh, UK. ${ }^{17}$ Alzheimer Scotland Dementia Research Centre, The University of Edinburgh, Edinburgh, UK. ${ }^{18}$ Rush Alzheimer's Disease Center, Rush University Medical Center, Chicago, IL, USA. ${ }^{19}$ Program in Translational NeuroPsychiatric Genomics, Department of Neurology, Brigham and Women's Hospital and Harvard Medical School, Boston, MA, USA. ${ }^{20}$ Rush Institute for Healthy Aging and Department of Internal Medicine, Rush University Medical Center, Chicago, IL, USA. ${ }^{21}$ Department of Epidemiology, Erasmus Medical Centre, Rotterdam, The Netherlands. ${ }^{22}$ Department of Neurology, Erasmus Medical Centre, Rotterdam, The Netherlands. ${ }^{23}$ Department of Radiology, Erasmus Medical Centre, Rotterdam, The Netherlands. ${ }^{24}$ Department of Child and Adolescent Psychiatry, Erasmus Medical Centre, Rotterdam, The Netherlands. ${ }^{25}$ Department of Psychiatry, Erasmus Medical Centre, Rotterdam, The Netherlands. ${ }^{26}$ Department of Internal Medicine, Erasmus Medical Centre, Rotterdam, The Netherlands. ${ }^{27}$ Interfaculty Institute for Genetics and Functional Genomics, University Medicine Greifswald, Greifswald, Germany. ${ }^{28}$ Institute for Community Medicine, University Medicine Greifswald, Greifswald, Germany. ${ }^{29}$ Department of Psychiatry and Psychotherapy, University Medicine Greifswald, HELIOS Hospital Stralsund, Greifswald, Germany. ${ }^{30}$ German Center for Neurodegenerative Diseases (DZNE), Site Rostock/Greifswald, Greifswald, Germany. ${ }^{31}$ Unit of Periodontology, Department of Restorative Dentistry, Periodontology and Endodontology, University Medicine Greifswald, Greifswald, Germany. ${ }^{32}$ Icelandic Heart Association, Kopavogur, Iceland. ${ }^{33}$ University of Iceland, Reykjavik, Iceland. ${ }^{34}$ National Institute on Aging, National Institutes of Health, Bethesda, MD, USA. ${ }^{35}$ Cardiovascular Health
Research Unit, Department of Medicine, University of Washington, Seattle, WA, USA. ${ }^{36}$ Translational Gerontology Branch, National Institute on Aging, Baltimore, MD, USA. ${ }^{37}$ Geriatric Unit, Azienda Sanitaria Firenze (ASF), Florence, Italy. ${ }^{38}$ Department of Epidemiology, Johns Hopkins Bloomberg School of Public Health, Baltimore, MD, USA. ${ }^{39}$ Division of Epidemiology and Community Health, University of Minnesota, Minneapolis, MN, USA. ${ }^{40}$ Department of Epidemiology, School of Public Health, University of North Carolina at Chapel Hill, Chapel Hill, NC 27514, USA.

Received: 22 July 2014 Accepted: 19 December 2014

Published online: 29 December 2014

\section{References}

1. Online Mendelian Inheritance in Man [http://www.omim.org/]

2. Hindorff LA, Sethupathy P, Junkins HA, Ramos EM, Mehta JP, Collins FS, Manolio TA: Potential etiologic and functional implications of genomewide association loci for human diseases and traits. Proc Natl Acad Sci U S A 2009, 106:9362-9367.

3. Mitton JB, Grant MC: Associations among protein heterozygosity, growth rate, and developmental homeostasis. Annu Rev Ecol Syst 1984, 15:479-499.

4. Alibert P, Renaud S, Dod B, Bonhomme F, Auffray JC: Fluctuating asymmetry in the Mus musculus hybrid zone: a heterotic effect in disrupted co-adapted genomes. Proc Biol Sci 1994, 258:53-59.

5. Roberts SC, Little AC, Gosling LM, Perrett DI, Carter V, Jones BC, Penton-Voak I, Petrie M: MHC-heterozygosity and human facial attractiveness. Evol Hum Behav 2005, 26:213-226.

6. Coetzee V, Barrett L, Greeff JM, Henzi SP, Perrett DI, Wadee AA: Common HLA alleles associated with health, but Not with facial attractiveness. PLoS One 2007, 2:e640.

7. Campbell H, Carothers AD, Rudan I, Hayward C, Biloglav Z, Barac L, Pericic M, Janicijevic B, Smolej-Narancic N, Polasek O, Kolcic I, Weber JL, Hastie ND Rudan P, Wright AF: Effects of genome-wide heterozygosity on a range of biomedically relevant human quantitative traits. Hum Mol Genet 2007, 16:233-241.

8. Takata H, Ishii T, Suzuki M, Sekiguchi S, Iri H: Influence of major histocompatibility complex region genes on human longevity among okinawan-japanese centenarians and nonagenarians. Lancet 1987, 330:824-826.

9. Lie HC, Simmons LW, Rhodes G: Does genetic diversity predict health in humans? PLoS One 2009, 4:e6391

10. Piertney SB, Oliver MK: The evolutionary ecology of the major histocompatibility complex. Heredity 2005, 96:7-21.

11. Charlesworth D, Willis JH: The genetics of inbreeding depression. Nat Rev Genet 2009, 10:783-796.

12. Szulkin M, Bierne N, David P: Heterozygosity-fitness correlations: a time for reappraisal. Evolution 2010, 64:1202-1217.

13. Arking DE, Chakravarti A: Understanding cardiovascular disease through the lens of genome-wide association studies. Trends Genet TIG 2009, 25:387-394.

14. Hindorff LA, Gillanders EM, Manolio TA: Genetic architecture of cancer and other complex diseases: lessons learned and future directions. Carcinogenesis 2011, 32:945-954.

15. Menard S: Six approaches to calculating standardized logistic regression coefficients. Am Stat 2004, 58:218-223.

16. Stouffer Samuel A, Suchman Edward A, DeViney Leland C, Star Shirley A, Williams Robin M Jr: The American Soldier. Adjusting During Army Life, Vol. 1. Princeton: Princeton University Press; 1949. 